\title{
Simultaneous or Scanning Data Acquisition? \\ A Theoretical Comparison Relevant \\ to Inductively Coupled Plasma Sector-Field \\ Mass Spectrometers
}

\author{
David A. Solyom and Gary M. Hieftje \\ Department of Chemistry, Indiana University, Bloomington, Indiana, USA
}

\begin{abstract}
The benefits of simultaneous multichannel detection over single-channel scanning detection are well established in analytical chemistry. Multichannel detection increases duty cycle, which leads to enhanced sensitivity, detection limits, and reduced analysis time. Also, multichannel detection used with either isotope-ratio or internal-standard techniques provides a mechanism to reduce the effect of multiplicative or flicker noise prevalent in plasma sources. An additional benefit of simultaneous detection is superior analysis of short-lived transient signals. Presented here is a theoretical comparison between simultaneous/continuous multichannel acquisition and single-channel scanning acquisition. To conduct this comparison, reported sensitivity, single-channel precision, and background values for commercial inductively coupled plasma sector-field mass spectrometers (ICP-SFMS) are used to generate theoretical figures of merit for both acquisition methods. Among the figures of merit that will be considered are detection limits, precision, and analysis time, particularly for multi-element or multi-isotope analysis. (J Am Soc Mass Spectrom 2003, 14, 227-235) (C) 2003 American Society for Mass Spectrometry
\end{abstract}

$\mathrm{M}$ ultichannel instruments are used to address a variety of analytical challenges including sample throughput, precision, and analysis time [1-3]. Also, multichannel acquisition can improve analysis of fast transient signals such as those produced by gas or liquid chromatography [4], flow-injection, capillary electrophoresis, electrothermal vaporization, and laser ablation. A variety of multichannel instruments for elemental analysis are commercially available today [5], the most prominent being multi-collector inductively coupled plasma sector-field mass spectrometers (ICP-SFMS) and ICP time-of-flight mass spectrometers (ICP-TOFMS) [6-8].

In addition to TOF and multi-collector ICP-SFMS instruments, the mass spectrograph [9] is a multichannel SFMS. A mass spectrograph, in contrast to conventional sector configurations, employs a multichannel detector array to examine continuously and simultaneously a broad mass range. Often, a geometry that produces a linear focal plane is desirable, so flat detector arrays can be used. The range of $m / z$ values that can be acquired simultaneously is limited by the size of the array relative to the dimensions and dispersion of the spectrograph. Theoretically, an entire atomic mass spec-

Published online February 24, 2003

Address reprint requests to: Professor Gary M. Hieftje, Indiana University, Department of Chemistry, Bloomington, IN 47405, USA. trum is obtainable if the array-detector size is not the limiting factor [10].

Commercial multi-collector instruments are equipped with a fixed number of Faraday cups, intended to measure isotopes of a single element. These detector elements are redistributed to match specific isotope patterns as needed. Typically, these multi-collector instruments are used in applications in which precision rather than elemental coverage or sensitivity is of paramount importance.

Despite the existence of multichannel instruments, the majority of commercial mass spectrometers are single-channel sequential scanning instruments [6]. As mentioned above, the multi-collector approach is reserved primarily for applications where a premium is placed on precision. Mass spectrographs using modern detector arrays are not commercially available, probably because of the significant limitations of array detectors suitable for mass spectrometry [11]. Existing array detectors fail to provide an adequate combination of gain, low noise, spatial resolution, and cost, among other figures of merit. Given the significant benefits of simultaneous data acquisition, development of such a detector would probably lead to the rapid commercialization of an array detector-based mass spectrograph system.

Presented here is a theoretical comparison of the fundamental differences between single-channel scan- 
ning acquisition and simultaneous, continuous multichannel acquisition. These differences will be applied to the performance levels of commercial ICP-SFMS instruments to project realistic benefits of multichannel acquisition for elemental analysis. The comparison will focus on multi-element analysis, measurement precision, the impact of Poisson and multiplicative source (or flicker) noise, and the integration time needed to achieve a specified signal-to-noise ratio. The terms simultaneous, continuous, and multichannel will all be used interchangeably. Conversely, scanning and singlechannel will be used in reference to conventional data acquisition.

The comparisons made here will deal strictly with mass spectrometer capabilities and not those of necessary auxiliary components. Omitted from this comparison is the impact of the detector/detection electronics and sample-introduction system on analytical performance. Therefore, the quantitative limitations of present mass spectrographic array detectors [11] will not be allowed to detract from the potential benefits to be realized through multichannel acquisition. Such limitations include restricted linear range, lack of uniform sensitivity among individual array elements, and variable noise characteristics among individual array elements. This analysis is justified on the basis of rapid recent developments in the array detector field [12, 13].

Also, the impact of trace impurities from the solvent blank, argon supply, air entrainment, and other possible sources of contamination will be disregarded. Considering the sensitivity and low continuum background noise of commercial ICP-SFMS instrumentation, these factors can elevate background levels significantly above the continuum floor. However, this limitation is a shortcoming of the components auxiliary to the mass spectrometer and plasma and will therefore be disregarded in our treatment. Because of its inherent nature, chemical noise such as argides produced by the ICP will be considered in specific treatments.

The reader should be cognizant of several specific circumstances where the theory presented here ceases to be consistent with experimental results. First, shortcomings in a data acquisition system can significantly limit the quality of acquired data. The gain provided by an electron multiplier is not uniform nor consistent with time, significantly limiting precision obtainable by a multiplier in analog mode relative to the capabilities of either Faraday cups in analog mode or counting-based detection with either an electron multiplier or microchannel plate(s).

Also, the linear range of the data acquisition system must be considered. With an ICP source, which generates on the order of $10^{8}$ counts s${ }^{-1} \mathrm{ppm}^{-1}$ for elements in solution, the upper counting-based limit of an electron multiplier linear range $\left(>10^{7} \mathrm{cps}\right)$ can be reached very easily. Beyond this limit, pulse pileup can limit accuracy, requiring the implementation of a correction factor [14]. Naturally, the consistency of this correction factor provides an additional quantitative barrier. The upper limit of the linear range is ordinarily not a concern with Faraday cup detection. Because the continuum background count rate in an ICP-MS typically exceeds the dark count rate of an electron multiplier, the lower end of the linear range is not limited by an electron multiplier or its accompanying detection electronics. The lower end of the linear range can be a concern with Faraday cup systems where Johnson noise in the readout electronics limits precision [15].

\section{Procedures, Boundary Conditions, and Assumptions}

\section{Duty Cycle and Scanning Rates}

All theoretical scanning data will be based on the assumption that the sample observation time is evenly distributed among all channels of interest. That is, the duty cycle (DC, see Table 1 for a definition of all terms and symbols) is the same for all channels. Time spent hopping and settling between masses will not be considered. Therefore, duty cycle will equal the reciprocal of the number of channels, $N$, or $D C=N^{-1}$. For example, a scanning instrument that monitors 50 different channels would exhibit a $2 \%$ duty cycle. Scanning rates are assumed to be slower than the dominant noise components in an ICP $[16,17]$ and, therefore, have no impact on ratio precision. Although this assumption might not be valid when short dwell times are used, it is valid when a large number of channels are being considered and conventional dwell times are used.

If the scanning rate in a sequential ICP-MS instrument were to exceed the predominant ICP-MS noise frequencies, the isotope-ratio precision of that instrument should agree with the Poisson-based statistical limit [18]. With the notable exception of ICP-TOFMS, all multi-channel ICP-MS instruments are assumed to provide $100 \%$ duty cycle. For ICP-TOFMS, duty cycle is inherently limited [7].

\section{Sensitivity}

Commercial ICP-SFMS instruments all possess a significant mass bias in favor of heavier elements. With conventional sample-introduction equipment and methods, typical ICP-SFMS sensitivities, $m_{x}$, range between $1 \times 10^{8}$ and $2 \times 10^{9} \mathrm{cps} / \mathrm{ppm}$. For the present study, sensitivity values of $1 \times 10^{8}, 1 \times 10^{9}$, and $2 \times 10^{9}$ $\mathrm{cps} / \mathrm{ppm}$ will be used. These values pertain to the Finnigan ELEMENT 2 (San Jose, CA) sensitivity specifications for lithium, indium, and uranium, respectively [19]. Initial discussions in the body of this work typically assume a concentration of $1 \mathrm{ppm}$ as a starting point, but the reader is encouraged to consider the impact of different concentrations and integration times on the derived equations presented here as they pertain to the reader's own interests. 
Table 1. Symbols, abbreviations, and corresponding terms appearing in text

\begin{tabular}{|c|c|}
\hline Symbol & Term \\
\hline$a_{i}$ & Analyte signal (cps) at a specified $\mathrm{m} / \mathrm{z}$ ratio $(\mathrm{i}=\mathrm{x}$ or $\mathrm{y})$ \\
\hline$b_{i}$ & Background continuum (cps) at specified $\mathrm{m} / \mathrm{z}(\mathrm{i}=\mathrm{x}$ or $\mathrm{y})$ \\
\hline $\mathrm{b}_{\mathrm{i}}^{\prime}$ & Background continuum (cps) at a specified $\mathrm{m} / \mathrm{z}(\mathrm{i}=\mathrm{x}$ or $\mathrm{y})$ in blank \\
\hline $\mathrm{c}_{\mathrm{i}}$ & Chemical background (cps) at a specified $\mathrm{m} / \mathrm{z}(\mathrm{i}=\mathrm{x}$ or $\mathrm{y})$ \\
\hline$c_{i}^{\prime}$ & Chemical background (cps) at a specified $\mathrm{m} / \mathrm{z}(\mathrm{i}=\mathrm{x}$ or $\mathrm{y})$ in blank \\
\hline DC & Duty cycle (unitless) \\
\hline f & Multiplicative noise designation \\
\hline LOD & Limit of detection (concentration) \\
\hline $\mathrm{m}_{\mathrm{x}}$ & Sensitivity of isotope at $\mathrm{m} / \mathrm{z} \times(\mathrm{cps} / \mathrm{ppm})$ \\
\hline $\mathrm{N}$ & Number of channels or $\mathrm{m} / \mathrm{z}$ values \\
\hline $\mathrm{P}$ & Poisson noise designation \\
\hline $\mathrm{R}$ & Ratio of analyte signals at $\mathrm{m} / \mathrm{z} x$ and $y\left(R=a_{x} / a_{y}\right)$ \\
\hline $\mathrm{S}$ & Signal strength (counts) \\
\hline $\mathrm{S} / \mathrm{N}$ & Signal-to-noise ratio (unitless) \\
\hline $\mathrm{T}$ & Total sample analysis time (seconds) \\
\hline $\mathrm{t}$ & Integration time for a specific channel (seconds) \\
\hline$[\mathrm{X}]$ & Concentration of analyte $X(p p m)$ \\
\hline $\mathrm{x}$ & First $\mathrm{m} / \mathrm{z}$ of interest \\
\hline $\mathrm{y}$ & Second $\mathrm{m} / \mathrm{z}$ of interest \\
\hline$\sigma$ & Standard deviation under specified conditions (signal type $(a, b$, or $c)$, noise type $(f$ or $P)$, and $\mathrm{m} / \mathrm{z}(\mathrm{x}$ or $\mathrm{y})$ ) \\
\hline$\sigma_{\mathrm{t}}$ & Total standard deviation at a specified $\mathrm{m} / \mathrm{z}$ ( $\mathrm{x}$ or $\mathrm{y}$ ) for a specified signal type $(\mathrm{a}, \mathrm{b}, \mathrm{or} \mathrm{c})$ \\
\hline$\sigma_{\mathrm{R}}$ & Standard deviation of the ratio, $\mathrm{R}$, of two $\mathrm{m} / \mathrm{z}$ values \\
\hline$\xi$ & Flicker factor [21] for $a, b$, and c (dimensionless) \\
\hline
\end{tabular}

\section{Signal Noise and Precision}

Typical ICP-SFMS signal precision ranges between $0.5 \%$ and $5.0 \%$ relative standard deviation (RSD), limited by multiplicative noise in the ICP and sample-introduction system $[16,20]$. The predominant frequencies of these noise components exceed the rates at which an ICP-SFMS can be scanned. Consequently, a single-channel system cannot ratio consecutive $\mathrm{m} / \mathrm{z}$ values as precisely as can a multichannel system. For a multichannel system, counting or Poisson statistics limits precision [21] because multiplicative source noise can be overcome through isotope-ratio [7] or internal-standardization techniques.

For the comparisons here, noise sources are separated into two categories: 1) noise that is multiplicative in nature and 2) noise dictated by Poisson statistics. Simulated single-channel results will include one of two different multiplicative source-noise levels, $1.0 \%$ and $0.2 \% \mathrm{RSD}$, in addition to counting statistics-related noise. Only noise due to counting statistics will be considered for theoretical multichannel data since multiplicative noise can then be overcome by ratioing [7]. A trio of isotope pairs, ${ }^{107,109} \mathrm{Ag},{ }^{20,22} \mathrm{Ne}$, and ${ }^{12,13} \mathrm{C}$, chosen because of their approximate abundance ratios, 1, 10, and 100 [22], respectively, will be used to generate ratio precision values.

\section{Continuum Background and Isobaric Interferences}

Commercial ICP-SFMS instruments produce a background continuum, $b$, in the $0.1-1.0 \mathrm{cps}$ range [23] in addition to chemical noise from significant isobaric interferences including but not limited to ${ }^{40} \mathrm{Ar}^{+},{ }^{40} \mathrm{Ar}^{2+}$, ${ }^{40} \mathrm{Ar}^{2+}$, and ${ }^{40} \mathrm{Ar}^{16} \mathrm{O}^{+}$. For all comparisons here, $b$ for both single- and multichannel instruments is assumed to be $1.0 \mathrm{cps}$ and constant across the entire atomic mass range. Also, chemical background or isobaric interferences, $c$, are included in specific calculations. The noise in $b$ and $c$ contains both a multiplicative component and a Poisson component for single-channel determinations, but the multiplicative component in both $b$ and $c$ is dropped for multichannel calculations. Further, it must be realized that the influence of chemical noise $c$ will depend on the sample concentrations that are employed. In turn, it will depend on whether ion counting with a multiplier detector or analog detection with a Faraday cup is utilized.

\section{Detection Limits}

The signal-to-noise ratio, $S / N$, for any technique is determined by a number of variables including sensitivity, background, background instability, signal variation, analyte concentration, and integration time. Conventionally, detection limits assume $D C=100 \%$, which is not realistic for multi-element or multi-isotopic applications. Detection limits for the two contrasting acquisition techniques will be plotted as a function of duty cycle for a range of total sample analysis times, $T$, where $T=t / D C$ and $t$ is integration time. Such a treatment reveals strong differences between the two approaches concerning effective sensitivity.

\section{Calculations and Equations}

The relationship between analyte signal, $a_{x}$, at a $m / z, x$, and the standard deviation of that signal due to multiplicative source (or flicker) noise, $\sigma_{a f x}$ is best described as a constant, $\xi$, sometimes termed the "flicker factor" $[21,24,25]$ : 


$$
\sigma_{a f x}=\xi \cdot a_{x}
$$

For simplicity, the flicker factor in this treatment is assumed to be constant and equal for all species types (analyte, background, and isobars) and $m / z$ values, but it should be noted that different sources of multiplicative noise can and do produce different $\xi$ values in actual experimental results. The standard deviation of the background continuum and chemical background due to multiplicative noise, $\sigma_{b f x}$ and $\sigma_{c f x}$, respectively, can be determined by substitution into eq 1 . Note that $\xi$ also represents the RSD (expressed as a fraction rather than a percentage) of a signal due to multiplicative source noise.

The noise from counting statistics on $a_{x}$ at $x, \sigma_{a P x}$, is an inherent aspect of counting-based detection and follows Poisson behavior [21]:

$$
\sigma_{a P x}=\sqrt{a_{x}}
$$

The background continuum and chemical noise due to counting statistics, $\sigma_{b P x}$ and $\sigma_{c P x}$, respectively, can also be determined by substitution into eq 2 .

Considering the additive nature of variances, the cumulative noise on $a_{x}, \sigma_{a t x}$, due to both flicker and Poisson noise is expressed as:

$$
\sigma_{a t x}=\sqrt{\sigma_{a f x}^{2}+\sigma_{a P x}^{2}}=\sqrt{\left(a_{x} \xi\right)^{2}+a_{x}}
$$

The total noise on the background continuum and chemical background signals, $\sigma_{b t x}$ and $\sigma_{c t x}$, respectively, can be determined by substitution into eq 3 . The total noise at $m / z x, \sigma_{t x}$, due to $a_{x}, b_{x}$, and $c_{x}$ can again be determined through the conventional additive treatment of variances:

$$
\begin{aligned}
\sigma_{t x} & =\sqrt{\sigma_{a t x}^{2}+\sigma_{b t x}^{2}+\sigma_{c t x}^{2}} \\
& =\sqrt{\left(a_{x} \xi\right)^{2}+a_{x}+\left(b_{x} \xi\right)^{2}+b_{x}+\left(c_{x} \xi\right)^{2}+c_{x}}
\end{aligned}
$$

Determination of the precision, $\sigma_{R}$, for a ratio, $R$, of the signals of two $m / z$ values, $x$ and $y\left(R=a_{x} / a_{y}\right)$, is significantly more complicated because $R$ is not simply determined by measurement of $a_{x}$ and $a_{y}$. Instead, $R$ is determined by measurement of the total signal at the two $m / z, x$ and $y\left(a_{x}+b_{x}+c_{x}\right.$ and $a_{y}+b_{y}+c_{y}$, respectively), followed by a blank correction $\left(b_{x}^{\prime}+c_{x}^{\prime}\right.$ and $b_{y}^{\prime}+c_{y}^{\prime}$, respectively). Symbolically, this function is expressed:

$$
R=\frac{\left(a_{x}+b_{x}+c_{x}\right)-\left(b_{x}^{\prime}+c_{x}^{\prime}\right)}{\left(a_{y}+b_{y}+c_{y}\right)-\left(b_{y}^{\prime}+c_{y}^{\prime}\right)}
$$

Propagation of uncertainty is necessary to develop the proper expression for $\sigma_{R}$ :

$$
\begin{aligned}
\sigma_{R}= & {\left[\left(\frac{\partial R}{\partial a_{x}}\right)^{2} \cdot \sigma_{a t x}^{2}+\left(\frac{\partial R}{\partial b_{x}}\right)^{2} \cdot \sigma_{b t x}^{2}\right.} \\
& +\left(\frac{\partial R}{\partial c_{x}}\right)^{2} \cdot \sigma_{c t x}^{2}\left(\frac{\partial R}{\partial b_{x}^{\prime}}\right)^{2} \cdot \sigma_{b^{\prime} t x}^{2}+\left(\frac{\partial R}{\partial c_{x}^{\prime}}\right)^{2} \cdot \sigma_{c^{\prime} t x}^{2} \\
& +\left(\frac{\partial R}{\partial a_{y}}\right)^{2} \cdot \sigma_{a t y}^{2}+\left(\frac{\partial R}{\partial b_{y}}\right)^{2} \cdot \sigma_{b t y}^{2}+\left(\frac{\partial R}{\partial c_{y}}\right)^{2} \cdot \sigma_{c t y}^{2} \\
& \left.+\left(\frac{\partial R}{\partial b_{y}^{\prime}}\right)^{2} \cdot \sigma_{b^{\prime} t y}^{2}+\left(\frac{\partial R}{\partial c_{y}^{\prime}}\right)^{2} \cdot \sigma_{c^{\prime} t y}^{2}\right]^{1 / 2}
\end{aligned}
$$

The partial derivatives are:

$$
\begin{aligned}
\frac{\partial R}{\partial a_{x}} & =\frac{\partial R}{\partial b_{x}}=\frac{\partial R}{\partial c_{x}}=-\frac{\partial R}{\partial b_{x}^{\prime}}=-\frac{\partial R}{\partial c_{x}^{\prime}} \\
& =\frac{1}{\left(a_{y}+b_{y}+c_{y}\right)-\left(b_{y}^{\prime}+c_{y}^{\prime}\right)} \\
\frac{\partial R}{\partial a_{y}} & =\frac{\partial R}{\partial b_{y}}=\frac{\partial R}{\partial c_{y}}=-\frac{\partial R}{\partial b_{y}^{\prime}}=-\frac{\partial R}{\partial c_{y}^{\prime}} \\
& =-\frac{\left(a_{x}+b_{x}+c_{x}\right)-\left(b_{x}^{\prime}+c_{x}^{\prime}\right)}{\left(\left(a_{y}+b_{y}+c_{y}\right)-\left(b_{y}^{\prime}+c_{y}^{\prime}\right)\right)^{2}}
\end{aligned}
$$

With the inclusion of the partial derivatives, eq 6 becomes:

$$
\begin{aligned}
\sigma_{R}= & \left(\frac{1}{\left(\left(a_{y}+b_{y}+c_{y}\right)-\left(b_{y}^{\prime}+c_{y}^{\prime}\right)\right)^{2}}\right. \\
& \cdot\left(\sigma_{a t x}^{2}+\sigma_{b t x}^{2}+\sigma_{c t x}^{2}+\sigma_{b^{\prime} t x}^{2}+\sigma_{c^{\prime} t x}^{2}\right) \\
& +\frac{\left(\left(a_{x}+b_{x}+c_{x}\right)-\left(b_{x}^{\prime}+c_{x}^{\prime}\right)\right)^{2}}{\left(\left(a_{y}+b_{y}+c_{y}\right)-\left(b_{y}^{\prime}+c_{y}^{\prime}\right)\right)^{4}} \\
& \left.\cdot\left(\sigma_{a t y}^{2}+\sigma_{b t y}^{2}+\sigma_{c t y}^{2}+\sigma_{b^{\prime} t y}^{2}+\sigma_{c^{\prime} t y}^{2}\right)\right)^{1 / 2}
\end{aligned}
$$

For the work here the continuum background, $b$, is assumed to be constant, which simplifies eq 8 . Also, the chemical noise (isobaric overlap) contributions, $c_{x}$ and $c^{\prime} x$, are assumed to be equal as are $c_{y}$ and $c^{\prime} y$. Based on these assumptions, other factors simplify as well:

$$
\begin{aligned}
& \sigma_{b t x}=\sigma_{b^{\prime} t x}=\sigma_{b t y}=\sigma_{b^{\prime} t y} ; \quad \sigma_{c t x}=\sigma_{c^{\prime} t x} ; \\
& \sigma_{c t y}=\sigma_{c^{\prime} t y}
\end{aligned}
$$

Therefore, eq 8 can be expressed as:

$$
\begin{aligned}
& \sigma_{R}= \\
& \sqrt{\frac{1}{a_{y}^{2}} \cdot\left(\sigma_{a t x}^{2}+2 \sigma_{b t}^{2}+2 \sigma_{c t x}^{2}\right)+\frac{a_{x}^{2}}{a_{y}^{4}} \cdot\left(\sigma_{a t y}^{2}+2 \sigma_{b t}^{2}+2 \sigma_{c t y}^{2}\right)}
\end{aligned}
$$




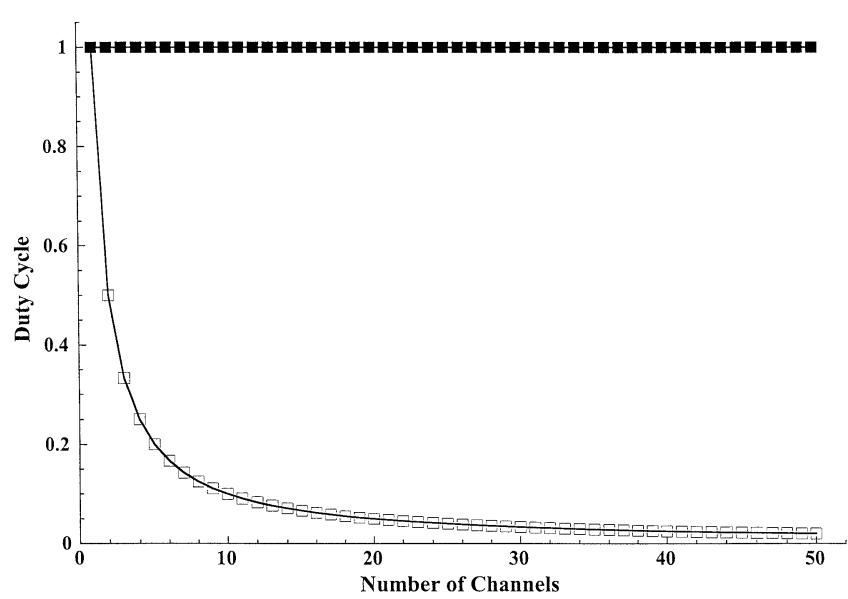

Figure 1. Duty cycle as a functionof total channels for both mul-

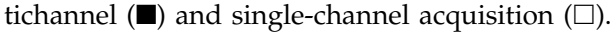

Replacing the six different standard deviations in eq 10 with their symbolic equivalents based on eq 3 will produce the final equation. This final form is cumbersome and, consequently, will not be included here. Conversion of eq 10 to an expression of the RSD of $R$ yields:

$$
\begin{aligned}
& \frac{\sigma_{R}}{R}= \\
& \sqrt{\frac{1}{a_{x}^{2}} \cdot\left(\sigma_{a t x}^{2}+2 \sigma_{b t}^{2}+2 \sigma_{c t x}^{2}\right)+\frac{1}{a_{y}^{2}} \cdot\left(\sigma_{a t y}^{2}+2 \sigma_{b t}^{2}+2 \sigma_{c t y}^{2}\right)}
\end{aligned}
$$

Theoretical detection limits will be calculated at a conventional $S / N$ of 3 with duty cycle, $D C$, also considered:

$$
\operatorname{LOD}(\mathrm{ppm})=3\left(\frac{\sqrt{b \cdot t}}{m_{x} \cdot t \cdot D C}\right)=3\left(\frac{\sqrt{b}}{m_{x} \cdot D C \cdot \sqrt{t}}\right)
$$

\section{Results and Discussion}

\section{Duty Cycle}

The most obvious benefit of multichannel acquisition is improved duty cycle. Figure 1 plots duty cycle for both single-channel and multichannel acquisition as a function of the number of channels. Duty cycle remains constant for a multichannel system but is inversely proportional to the number of channels for scanning acquisition. Since effective sensitivity is directly proportional to duty cycle, the same difference in effective sensitivity is found between the two methods. As cited earlier in the text, ICP-TOFMS is a notable exception in this comparison between the duty cycles of sequential and simultaneous instruments. While the duty cycle of an ICP-TOFMS instrument is independent of the number of channels of interest, it is inherently limited in duty cycle to a value well below $100 \%$ (5-10\% is typical) [7].

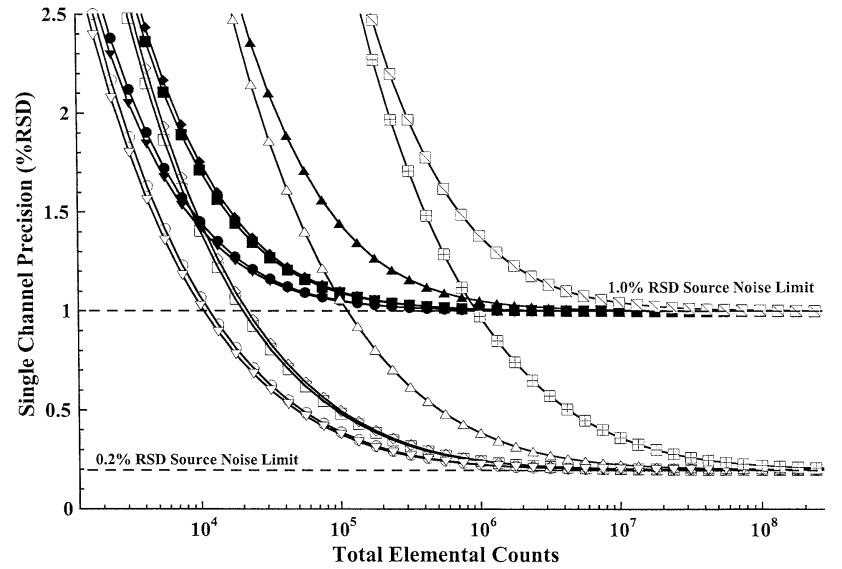

Figure 2. Non-ratioed signal precision for three different isotope pairs as a function of total elemental counts. Multiplicative source noise (flicker) for each plot is either $1.0 \%$ or $0.2 \%$ RSD (see legend). Note that the $\mathrm{X}$-axis is logarithmic.

- ${ }^{107} \mathrm{Ag} 1.0 \%$ Flicker Noise

$\boldsymbol{} \overrightarrow{ }{ }^{109} \mathrm{Ag} 1.0 \%$ Flicker Noise

$\square={ }^{107} \mathrm{Ag} 0.2 \%$ Flicker Noise

$={ }^{20} \mathrm{Ne} 1.0 \%$ Flicker Noise

$\diamond={ }^{109} \mathrm{Ag} 0.2 \%$ Flicker Noise

$\boldsymbol{\Delta}={ }^{22} \mathrm{Ne} 1.0 \%$ Flicker Noise

$\mathrm{O}={ }^{20} \mathrm{Ne} 0.2 \%$ Flicker Noise

$\boldsymbol{\nabla}={ }^{12} \mathrm{C} 1.0 \%$ Flicker Noise

$\triangle={ }^{22} \mathrm{Ne} 0.2 \%$ Flicker Noise

$\nabla={ }^{13} \mathrm{C} 1.0 \%$ Flicker Noise

$\nabla={ }^{12} \mathrm{C} 1.0 \%$ Flicker Noise

$\boxplus={ }^{13} \mathrm{C} 1.0 \%$ Flicker Noise

Figure 1 also reveals problems for each acquisition technique. The fraction of analyte that is wasted by the scanning system increases with the number of channels and equals $1-D C$. On the other hand, the $100 \%$ duty cycle of a multichannel system requires data to be registered at a much faster rate. An array detectorbased detection system must process data in a more sophisticated fashion than a single-channel detection system.

A single-channel ICP-SFMS instrument that uses an electron multiplier and counting-based acquisition processes only one count at a time. Further, the average time between counts must be at least 10 times greater than the dead time of the detection electronics to avoid serious pulse pileup. Because a multichannel system can acquire a much greater amount of data simultaneously, it is decidedly superior. This combination makes single-channel operation relatively time-consuming if many isotopes are to be measured. However, a suitable array detection system must either possess many parallel counting channels or, if multiplexed, must operate on an extremely fast time scale. Alternatively, an array detector that accumulates and integrates the signal for an extended period of time (0.01-100 seconds) before reading data to storage, in a manner analogous to charge-transfer devices used in emission spectrometry [26], would be desirable.

\section{Single-Channel Signal Precision}

Figure 2 shows non-ratioed signal precision (\% RSD) for three different isotope pairs, ${ }^{107,109} \mathrm{Ag},{ }^{20,22} \mathrm{Ne}$, and ${ }^{12,13} \mathrm{C}$, as a function of accumulated counts. Note that the $\mathrm{X}$-axis is a logarithmic scale and that two alternative 
values for multiplicative source noise (flicker), 1.0\% $(\xi=0.01)$ and $0.2 \% \operatorname{RSD}(\xi=0.002)$, are included. The assumed elemental concentration is $1 \mathrm{ppm}$ for each element. At this concentration range, the influence of chemical background and background continuum on analyte precision was assumed to be negligible and was therefore disregarded (see eqs 3 and 4 ).

A number of points are readily apparent in Figure 2. First, flicker noise renders extended integration times fruitless. With multiplicative source noise at 1.0\% RSD, no more than $10^{5}$ total elemental counts are worth accumulating for isotopes with relative abundances approaching $100 \%\left({ }^{20} \mathrm{Ne},{ }^{12} \mathrm{C}\right)$ before the source noise becomes precision-limiting. Because of the 100 -fold disparity in relative abundance, isotopes with only $1 \%$ relative abundance $\left({ }^{13} \mathrm{C}\right)$, require accumulation of a 100 -fold greater number of total elemental counts $\left(10^{7}\right.$ counts) to reach the multiplicative source-noise limit. Considering the sensitivity of commercial ICP-SFMS instruments, very short integration periods are adequate to accumulate this many counts. Even for a $1 \mathrm{ppb}$ solution, isotopes of $1 \%$ relative abundance require only a 10-second integration time.

Reducing multiplicative source noise to $0.2 \%$ RSD extends the necessary number of accumulated counts to reach the source-noise limit. For isotopes with a relative abundance approaching $100 \%$, roughly $10^{7}$ total elemental counts are necessary before the signal is sourcenoise limited $\left(10^{9}\right.$ counts for isotopes with $1 \%$ relative abundance). Again, considering the sensitivity of commercial ICP-SFMS instruments, relatively short integration periods and moderate analyte concentrations are sufficient to reach this limit.

\section{Isotope-Ratio Precision}

Figure 3 displays single-channel and multichannel isotope-ratio precision by plotting ratio \%RSD as a function of total elemental counts (see eq 11). The same three pairs of isotopes considered in Figure 2 are used. \%RSD values for scanning-based ratios were derived on the basis of two different flicker noise levels, $1.0 \%$ and $0.2 \%$ RSD, for the individual channels. In addition to these six plots, a final set of three traces shows ratio precision for multichannel acquisition where contributions from flicker noise have been disregarded. A background count rate of $1.0 \mathrm{cps}$ was included but no chemical background was added.

Just as with single-channel signal precision, singlechannel isotope-ratio precision is limited by flicker noise. Examination of eq 11 indicates that isotope-ratio precision is governed by the precision of both individual signals that comprise the ratio. Under conditions where flicker noise is limiting, both isotopes experience noise of equal $\%$ RSD (either $1.0 \%$ or $0.2 \%$ for this discussion). As a result, flicker noise limits isotope-ratio precision for single-channel acquisition to the precision limit of an individual channel $(1.0 \%$ or $0.2 \%)$ multiplied by $2^{1 / 2}$.

A closer look at Figures 2 and 3 shows there is a

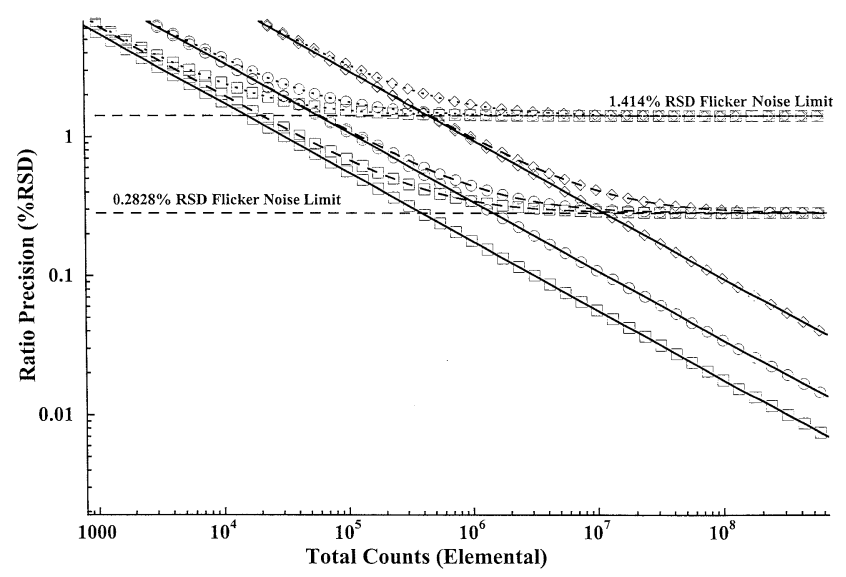

Figure 3. Isotope-ratio \%RSD for three different isotope pairs as a function of total elemental counts. Three sets of three ratios are shown. Two are for ratio precision with scanning acquisition and either $1.0 \%$ or $0.2 \%$ source flicker noise. The third set is for ratio precision by simultaneous acquisition and is independent of source flicker noise. Note that both axes are logarithmic.

$\cdots \cdots \cdots \cdots \cdot={ }^{107,109} \mathrm{Ag}$ ratio, Scanning acquisition, $1.0 \%$ source flicker ........... $={ }^{20,22} \mathrm{Ne}$ ratio, Scanning acquisition, $1.0 \%$ source flicker $\cdots . . . \cdots .={ }^{12,13} \mathrm{C}$ ratio, Scanning acquisition, $1.0 \%$ source flicker

- - - - $={ }^{107,109} \mathrm{Ag}$ ratio, Scanning acquisition, $0.2 \%$ source flicker

- $\Theta-\cdot={ }^{20,22} \mathrm{Ne}$ ratio, Scanning acquisition, $0.2 \%$ source flicker

$-\diamond--={ }^{12,13} \mathrm{C}$ ratio, Scanning acquisition, $0.2 \%$ source flicker

$\square={ }^{107,109} \mathrm{Ag}$ ratio, Simultaneous acquisition

$-{ }^{20,22} \mathrm{Ne}$ ratio, Simultaneous acquisition

$\diamond={ }^{12,13} \mathrm{C}$ ratio, Simultaneous acquisition

direct relationship between the number of elemental counts necessary for the ratio precision to reach its flicker-noise limit and the number of elemental counts necessary for the single-channel precision of the less abundant isotope in the ratio $\left({ }^{109} \mathrm{Ag},{ }^{22} \mathrm{Ne}\right.$, or ${ }^{13} \mathrm{C}$ in this presentation) to reach its flicker-noise limit. This correlation is expected since the less abundant isotopes will require longer integration times to generate enough counts so counting-statistics noise becomes insignificant.

The traces pertaining to simultaneous acquisition in Figure 3 show that only the number of accumulated signal counts limits ratio precision in a multichannel system. For ICP-SFMS instruments that possess high sensitivity, extraordinarily high precision is possible. With only $10^{8}$ total elemental counts acquired, precision for the three pairs of isotopes ranges from a high of $0.09 \%$ RSD for ${ }^{12} \mathrm{C} /{ }^{13} \mathrm{C}$ to a low of $0.02 \%$ RSD for ${ }^{107} \mathrm{Ag} /{ }^{109} \mathrm{Ag}$. With each order of magnitude increase in signal accumulation, precision improves by $10_{1 / 2}^{1 / 2}$. Therefore, accumulation of $10^{10}$ counts will yield precision better than $0.01 \%$ RSD for virtually any isotopic pair desired. With the sensitivity of existing ICP-SFMS instrumentation, such levels can be reached with $1 \mathrm{ppm}$ samples and 10-second integration times. The reader should keep in mind that Johnson noise becomes an issue in this regime [15].

\section{Chemical Background}

Figure 4 illustrates the impact that a high chemical background will have on measurement precision for 


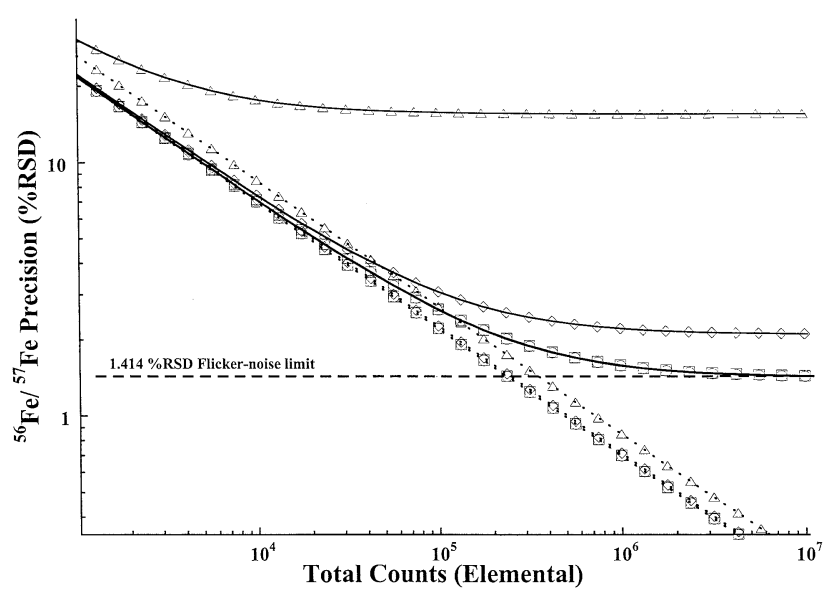

Figure 4. Ratio precision (\%RSD) for ${ }^{56} \mathrm{Fe} /{ }^{57} \mathrm{Fe}$ in the presence of significant chemical background $\left({ }^{40} \mathrm{ArO}^{+}\right)$and $1.0 \%$ flicker noise. Iron concentration and sensitivity are assumed to be $1 \mathrm{ppm}$ and $10^{9} \mathrm{cps} / \mathrm{ppm}$, respectively, and the background is $1 \mathrm{cps}$. Four different ${ }^{40} \mathrm{ArO}^{+}$signal strengths, $0,10^{8}, 10^{9}$, and $10^{10} \mathrm{cps}$ are included with both scanning and simultaneous acquisition. Note that both axes are logarithmic in scale.

- $=$ Scanning acquisition, no ${ }^{40} \mathrm{ArO}^{+}$background

$\square=$ Scanning acquisition, $10^{8} \mathrm{cps}{ }^{40} \mathrm{ArO}^{+}$background

$\curlyvee=$ Scanning acquisition, $10^{9} \mathrm{cps}^{40} \mathrm{ArO}^{+}$background

$\widehat{\triangle}=$ Scanning acquisition, $10^{10} \mathrm{cps}{ }^{40} \mathrm{ArO}^{+}$background

$\cdots \odot \cdots=$ Simultaneous acquisition, no ${ }^{40} \mathrm{ArO}^{+}$background

...... = Simultaneous acquisition, $10^{8} \mathrm{cps}{ }^{40} \mathrm{ArO}^{+}$background

... $\leftrightarrow \cdots=$ Simultaneous acquisition, $10^{9} \mathrm{cps}{ }^{40} \mathrm{ArO}^{+}$background

.... $. \cdots=$ Simultaneous acquisition, $10^{10} \mathrm{cps}^{40} \mathrm{ArO}^{+}$background

ratios obtained with either a single-channel or multichannel instrument (see eq 11). For this study, an ${ }^{40} \mathrm{ArO}^{+}$chemical background is added to the signal at ${ }^{56} \mathrm{Fe}$ and its effect on the ${ }^{56} \mathrm{Fe} /{ }^{57} \mathrm{Fe}$ ratio precision (a 1 ppm Fe solution and an Fe sensitivity of $10^{9} \mathrm{cps} / \mathrm{ppm}$ are assumed) is determined. Four different strengths of ${ }^{40} \mathrm{ArO}^{+}, 0,10^{8}, 10^{9}$, and $10^{10} \mathrm{cps}$, were chosen and considered in both a simultaneous and scanning instrument with $1.0 \%$ RSD flicker noise.

With no chemical background present, the ratio precision asymptotically approaches $1.414 \%$ RSD with single-channel scanning acquisition, in agreement with Figure 3. Even at fairly high levels of chemical background $\left(10^{8} \mathrm{cps}\right)$, the ratio precision does not significantly deviate from the behavior observed in the absence of the background. However, when the ${ }^{40} \mathrm{ArO}^{+}$ signal approaches that of the analyte with which it interferes, the precision limit is affected. With the analyte and chemical background at equal strength (each $10^{9} \mathrm{cps}$ ), the precision limit is elevated $60 \%$ to above $2 \%$ RSD. As the chemical background becomes even larger relative to the analyte signal, the chemical background flicker noise becomes the determining factor for ratio precision.

In the case of simultaneous detection, the impact of chemical background is less severe due to the removal of the flicker noise components from eq 11. Only the most extreme case of chemical noise presented in Figure $4,10^{10} \mathrm{cps}{ }^{40} \mathrm{ArO}^{+}$, produced a significant deviation from results expected in the absence of chemical noise. Furthermore, the flicker noise in the chemical back-

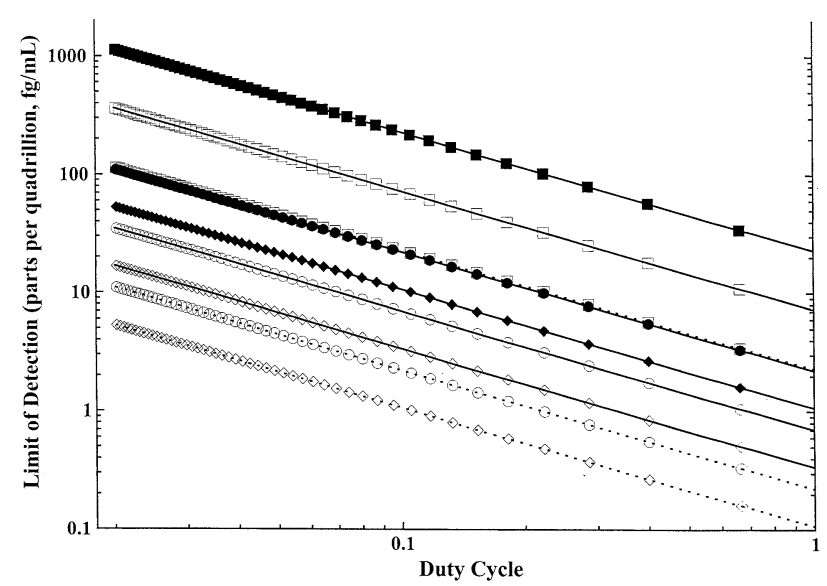

Figure 5. Effect of duty cycle on detection limits for ${ }^{7} \mathrm{Li},{ }^{115} \mathrm{In}$, and ${ }^{238} \mathrm{U}$ at three total sample analysis times, 2, 20, and 200 seconds. Note that the 2 -second ${ }^{115} \mathrm{In}$ trace and the 200 -second ${ }^{7} \mathrm{Li}$ trace overlap significantly and that both axes are logarithmic in scale.

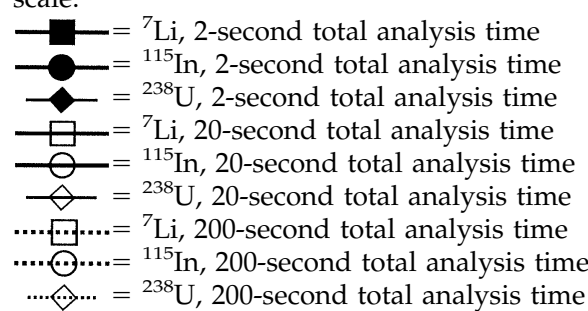

ground no longer limits the obtainable ratio precision. Instead, the precision is elevated a fixed amount, no matter the number of recorded counts.

This simulation was carried out by assuming a high elemental concentration and an intense chemical background. However, similar results would be obtained with weaker intense signals as long as the relative proportions of the analyte and chemical background are maintained.

\section{Detection Limits}

ICP-MS instrumentation is capable of providing low detection limits in a multi-element mode for most elements in the periodic table. However, for the method to realize this capability, the time needed to reach a desired $S / N$ for the entire elemental mass spectrum must be considered. Here is another area where the ability to acquire data simultaneously and continuously is beneficial.

Figure 5 shows detection limits for ${ }^{7} \mathrm{Li},{ }^{115} \mathrm{In}$, and ${ }^{238} \mathrm{U}$ as a function of duty cycle (see eq 12). The background count rate is assumed to be $1 \mathrm{cps}$. For these determinations, three different total sample analysis times, 2, 20, and 200 seconds, are considered. Note that both axes of Figure 5 are logarithmic scale. Detection limits in a multichannel environment correspond simply to the values plotted at $D C=100 \%$, while detection limits for a single-channel instrument correspond to the achievable duty cycle.

The shortest analysis time, 2 seconds, is similar in duration to many types of transient signals. Assuming a 
duty cycle of $100 \%$, ${ }^{7} \mathrm{Li}$ detection limits are extremely low $(23 \mathrm{fg} / \mathrm{mL}$ or ppq) for this analysis time. However, a single-channel instrument with a duty cycle of $2 \%$ results, as expected, in a 50-fold worsening of detection limits to slightly above $1 \mathrm{pg} / \mathrm{mL}$ (pptr). Also with a two-second analysis time, detection limits for ${ }^{115} \mathrm{In}$ and ${ }^{238} \mathrm{U}$ with $D C=100 \%$ are 2.3 and $1 \mathrm{ppq}$, respectively. Analogous values at a $2 \%$ duty cycle are 110 and 50 ppq, respectively. These values are still excellent and suitable for many applications but, nonetheless, are significantly inferior to the multichannel approach. With extended analysis times, both ${ }^{238} \mathrm{U}$ and ${ }^{115} \mathrm{In}$ detection limits are better than $1 \mathrm{ppq}$ and ${ }^{7} \mathrm{Li}$ limits are roughly $10 \mathrm{ppq}$, assuming high duty cycle. The reader should keep in mind that these values should be considered theoretical only as great difficulty is encountered in dealing with analytes in the ppq to low pptr range.

Analysis time can obviously be lengthened to improve detection limits, but temporal resolution is compromised. Also, the square root relationship between integration time and detection limits means that a 100 -fold increase in integration time is necessary to realize a full order of magnitude improvement in detection limits. Clearly, increasing duty cycle is a more attractive method of lowering detection limits.

\section{Conclusions}

Significant calculated differences between multichannel and single-channel acquisition have been presented by using operating parameters typical for modern ICPSFMS instrumentation. Simultaneous detection directly improves precision and duty cycle, which, in turn, raises sensitivity, aids transient signal analysis, and simplifies analysis of samples that are either restricted in size or possess a dynamic chemical composition.

Simultaneous detection is a mechanism for overcoming multiplicative source noise (flicker), which limits precision in conventional ICP-MS instruments to the $0.5-5.0 \%$ RSD range. By using simultaneous detection in conjunction with isotope-ratio or internal standardization-techniques, measurement precision becomes limited solely by counting statistics, not by flicker noise. Because of the high sensitivity provided by ICP-SFMS instrumentation, the counting-statistics limit can conceivably be pushed below $0.01 \%$ RSD with reasonable analyte concentrations and integration periods.

An instrument that features simultaneous detection inherently offers $100 \%$ duty cycle, increasing the effective sensitivity for the analysis of complex multi-element solutions. This higher sensitivity reduces the necessary analysis time to reach a specified $S / N$ level. In turn, this capability can translate to less sample consumption, reduced analysis time, lower detection limits, or a combination of all three.

Scanning instruments are known to have difficulty representing transient signals such as chromatographic peaks with high quantitative integrity. The ability to reduce analysis time has been established here as a benefit of simultaneous acquisition. Therefore, the analysis of transient signals, inherently limited in duration, is executed more accurately with simultaneous and continuous data acquisition.

\section{Acknowledgments}

Supported in part by the U.S. Department of Energy through the Office of Nonproliferation Research and Engineering, contract \#40159-A9E.

\section{References}

1. Bayliss, M. K.; Little, D.; Mallett, D. N.; Plumb, R. S. Parallel ultra-high flow rate liquid chromatography with mass spectrometric detection using a multiplex electrospray source for direct, sensitive determination of pharmaceuticals in plasma at extremely high throughput. Rapid Commun. Mass Spectrom. 2000, 14, 2039-2045.

2. Lewis, E. N.; Treado, P. J.; Reeder, R. C.; Story, G. M.; Dowrey, A. E.; Marcott, C.; Levin, I. W. Fourier Transform Spectroscopic Imaging Using an Infrared Focal-Plane Array Detector. Anal. Chem. 1995, 67, 3377-3381.

3. Rehkamper, M.; Mezger, K. Investigation of matrix effects for $\mathrm{Pb}$ isotope ratio measurements by multiple collector ICP-MS: verification and application of optimized analytical protocols. J. Anal. At. Spectrom. 2000, 15, 1451-1460.

4. Holland, J. F.; Enke, C. G.; Allison, J.; Stults, J. T.; Pinkston, J. D.; Newcome, B.; Watson, J. T. Mass Spectrometry on the Chromatographic Time Scale: Realistic Expectations. Anal. Chem. 1983, 55, 997A-1112A.

5. Schneider, C. A. An entry-level ICP with scanning CCD detector. Am. Lab. 2000, 32, 72-74.

6. Erickson, B. E. ICPMS-Beyond quadrupole. Anal. Chem. 1999, $71,811 \mathrm{~A}-815 \mathrm{~A}$.

7. Mahoney, P. P.; Ray, Steven J.; Hieftje, Gary M. Time-of-Flight Mass Spectrometry for Elemental Analysis. Appl. Spectrosc. 1997, 51, 16A-28A.

8. Guilhaus, M. Essential elements of time-of-flight mass spectrometry in combination with the inductively coupled plasma ion source. Spectrochim. Acta, Part B 2000, 55B, 1511-1525.

9. Todd, J. F. J. Recommendations for Nomenclature and Symbolism for Mass Spectrometry. Int. J. Mass Spectrom. Ion Processes 1995, 142, 211-240.

10. Cromwell, E. F.; Arrowsmith, P. Novel multichannel plasmasource mass spectrometer. J. Am. Soc. Mass Spectrom. 1996, 7 , $458-466$.

11. Birkinshaw, K. Fundamentals of focal plane detectors. J. Mass Spectrom. 1997, 32, 795-806.

12. Denton, M. B. "Advances in the application of array detectors for improved chemical analysis", 27th Annual Conference of the Federation of Analytical Chemistry and Spectroscopy Societies, Paper \#283, Nashville, TN, 2000.

13. Koppenaal, D.; Barinaga, C. J. Focal plane detectors in atomic mass spectrometry - past/present/future, 27th Annual Conference of the Federation of Analytical Chemistry and Spectroscopy Societies, Paper \#311, Nashville, TN, 2000.

14. Nelms, S. M.; Quétel, C. R.; Prohaska, T.; Vogl, J.; Taylor, D. P. Evaluation of detector dead time calculation models for ICPMS. J. Anal. At. Spectrom. 2001, 16, 333-338.

15. "TRITON NEPTUNE, multicollector mass spectrometers for high precision isotope ratio determination," http://www. thermo.com/eThermo/CMA/PDFs/Product/ productPDF_14630.pdf.

16. Furuta, N.; Monnig, C. A.; Yang, P.; Hieftje, G. M. Noise characteristics of an inductively coupled plasma-mass spectrometer. Spectrochim. Acta, Part B 1989, 44B, 649-656. 
17. Furuta, N. Optimization of the mass scanning rate for the determination of lead isotope ratios using an inductively coupled plasma mass spectrometer. J. Anal. At. Spectrom. 1991, 6, 199-203.

18. Bandura, D. R.; Baranov, V. I.; Tanner, S. D. Effect of collisional damping and reactions in a dynamic reaction cell on the precision of isotope ratio measurements. J. Anal. At. Spectrom. 2000, 15, 921-928.

19. Thermo homepage, http://www.thermo.com.

20. Ince, A. T.; Williams, J. G.; Gray, A. L. Noise in inductively coupled plasma mass spectrometry: Some preliminary measurements. J. Anal. At. Spectrom. 1993, 8, 899-903.

21. Skoog, D.A.; Leary, J.J. Principles of Instrumental Analysis, 4th ed.; Harcourt Brace Jovanovich: Orlando, FL, 1992.

22. Rosman, K. J. R.; Taylor, P. D. P. Isotopic compositions of the elements 1997. J. Anal. At. Spectrom. 1999, 14, 5N-24N.
23. Quetel, C. R.; Vogl, J.; Prohaska, T.; Nelms, S.; Taylor, P. D. P.; De Bievre, P. Comparative performance study of ICP mass spectrometers by means of $U$ "isotopic measurements." Fresenius' J. Anal. Chem. 2000, 368, 148-155.

24. Alkemade, C. T. J.; Snelleman, W.; Boutilier, G. D.; Pollard, B. D.; Winefordner, J. D.; Chester, T. L.; Omenetto, N. A review and tutorial discussion of noise and signal-to-noise ratios in analytical spectrometry-I. Fundamental principles of signal-tonoise ratios. Spectrochim. Acta, Part B 1978, 33B, 383-399.

25. Winefordner, J. D.; Parsons, M. L.; Mansfield, J. M.; McCarthy, W. J. Derivation of expressions for calculation of limiting detectable atomic concentration in atomic fluorescence flame spectrometry. Anal. Chem. 1967, 39, 436-442.

26. Hanley, Q. S.; Earle, C. W.; Pennebaker, F. M.; Madden, S. P.; Denton, M. B. Charge-transfer devices in analytical instrumentation. Anal. Chem. 1996, 68, 661A-667A. 